magnitude. There were inverse associations between adult depression/anxiety with sports/recreational activity $(-0.06)$ and activity at work $(-0.07)$. Lower educational level was associated with a higher level of activity at work (0.31) and a lower level of sports/ recreational activity $(-0.12)$.

Conclusions We found that the effect of childhood socio-economic adversity on adult physical activity was entirely mediated by educational attainment. The association between adolescent depression/anxiety and activity was entirely mediated by current mental health problems. These results highlight the importance of education in reducing the adverse effect of childhood socioeconomic conditions on adult physical activity. Furthermore, addressing current mental health status should be seen as a priority for policies aimed at physical activity in adulthood.

\section{PHYSICAL ACTIVITY ACROSS ADULTHOOD AND PHYSICAL CAPABILITY IN MID-LIFE: FINDINGS FROM A BRITISH BIRTH COHORT STUDY}

doi:10.1136/jech.2010.120956.70

R Cooper, G Mishra, D Kuh. MRC Unit for Lifelong Health and Ageing and Division of Population Health, University College London, London, UK

Background Maintaining high levels of physical capability with age is important given that low levels are associated with increased risk of losing independence, health problems and mortality. Studies of older people provide evidence to suggest that physical activity may be beneficial for the maintenance of physical capability however it is unclear whether the effects of physical activity accumulate over the lifecourse.

Objectives To test the associations between physical activity levels, assessed by self-report of participation in sports and recreational activities prospectively at three ages across adulthood (36, 43 and 53 years), and objective measures of physical capability at age 53 year; to examine whether any associations found are independent of physical activity levels at other ages and other potential confounders.

Design Prospective cohort study.

Setting England, Scotland and Wales.

Participants Approximately 2400 men and women from the MRC National Survey of Health and Development, followed up since birth in March 1946.

Main outcome measures Grip strength, standing balance and chair rise time assessed by nurses during home visits at age 53 year.

Results Physical activity levels at all three ages in adulthood were positively associated with chair rise and standing balance performance. These associations were maintained after adjustment for sex, height, weight and socio-economic position with those people who were categorised as being most active performing better in these two tests than people reporting no activity. In models which included physical activity at all three ages simultaneously, there was evidence of independent positive effects of participation in sports and recreational activities at all three ages on chair rise performance and at ages 43 and 53 years on standing balance performance. Differences in mean chair rise time $(1 /$ time $(s) \times 100)$ between the most active and least active groups were: (at age 53 year: $0.30(95 \%$ CI 0.14 to 0.46$)$; at ages 36 and 43 year: $0.36(0.18,0.54)$ ) after adjustment for activity levels at the other two ages and covariates. There was no evidence of associations between physical activity levels at any age and grip strength in women and in men only physical activity at age 53 year was associated with grip strength. Conclusions Evidence of independent effects of physical activity at different ages across adulthood on chair rise and standing balance performance in mid-life suggests that there are cumulative benefits of physical activity across adulthood for physical capability in midlife. Increased activity should therefore be promoted earlier in life. 071 DIFFERENT EFFECTS OF BODY MASS INDEX AND PHYSICAL
ACTIVITY ON THE RISK OF ANKLE, WRIST AND HIP FRACTURES IN POSTMENOPAUSAL WOMEN

doi:10.1136/jech.2010.120956.71

M E G Armstrong, E A Spencer, B J Cairns, V Beral, for the Million Women Study Collaborators. Cancer Research UK Epidemiology Unit, University of Oxford, Oxford, UK

Objective To compare the relationship between body mass index (BMI) and physical activity for the risk of ankle, wrist and hip fractures in a large prospective study of postmenopausal women in the UK.

Methods In 1996-2001, women recruited into the Million Women Study completed a self-administered questionnaire asking about body size, physical activity, disease history, and lifestyle and reproductive factors. Incident ankle, wrist and hip fractures were identified through self-report in a follow-up questionnaire completed an average of 3.2 years after recruitment. RRs and CIs for each fracture site in postmenopausal women by BMI and physical activity at recruitment were calculated using Cox regression models, adjusted for socio-economic status, smoking and other health and lifestyle factors.

Results Among 599550 postmenopausal women, 5114 reported an ankle fracture, 8565 reported a wrist fracture, and 754 reported a hip fracture during follow-up. When compared to lean women $\left(\mathrm{BMI}<22.5 \mathrm{~kg} / \mathrm{m}^{2}\right)$, obese women $\left(\mathrm{BMI} \geq 30 \mathrm{~kg} / \mathrm{m}^{2}\right)$ had an increased risk of ankle fracture (RR 2.47; 95\% CI 2.32 to to 2.63), but a decreased risk of wrist fracture (RR $0.68 ; 95 \%$ CI 0.64 to to 0.72 ) and hip fracture (RR $0.29 ; 95 \%$ CI 0.23 to to 0.37$)\left(\chi^{2}<0.001\right.$ for the effect of BMI for each fracture site). Physical activity had little influence on the risk of either ankle fracture or wrist fracture, but women who reported strenuous physical activities up to once per week or more often were at a lower risk of hip fracture than women who reported less frequent activity (RR 0.65 ; 95\% CI 0.56 to to $0.76)$.

Conclusions BMI and physical activity have different effects on the incidence of fracture at different sites. While obese women are at increased risk of ankle fracture they are at lower risk of wrist fracture and hip fracture. Physical activity has no marked influence on ankle and wrist fracture but is protective against hip fracture.

\section{Adolescence}

072 UNDERSTANDING SOCIAL AND CULTURAL INFLUENCES ON THE RELATIONSHIPS AND SEXUAL EXPERIENCES OF YOUNG BRITISH PAKISTANIS IN LONDON: IS THERE UNMET SEXUAL HEALTH NEED?

doi:10.1136/jech.2010.120956.72

${ }^{1} \mathrm{C}$ Griffiths, ${ }^{2} \mathrm{~A}$ Prost, ${ }^{1} \mathrm{G}$ J Hart. ${ }^{1}$ Centre for Sexual Health and HIV Research, Department of Infection and Population Health, University College London, London, UK ${ }^{2}$ Centre for International Health and Development, University College London, London, UK

Background and aim Health services should take account of cultural and faith diversity. Pakistanis are the UKs second largest ethnic group and one of the largest Muslim communities. However, relative to other ethnic minority groups, there is a paucity of sexual health research among this group. Using community-based qualitative research we explored the social and cultural influences on sexual attitudes and experiences of young Pakistanis in East London to determine whether there is unmet sexual health need and implications for service development.

Design Between June and September 2008, 30 in-depth one-to-one interviews (60-90 min) were conducted with young Pakistanis 